\title{
On the angular dependence of focused laser ablation by nanosecond pulses in solgel and polymer materials
}

\author{
D. S. George, A. Onischenko, and A. S. Holmes ${ }^{\text {a) }}$ \\ Optical \& Semiconductor Devices Group, Department of Electrical \& Electronic Engineering, \\ Imperial College London, Exhibition Road, London, SW7 2BT United Kingdom
}

(Received 14 October 2003; accepted 7 January 2004)

\begin{abstract}
Focused laser ablation by single laser pulses at varying angles of incidence is studied in two materials of interest: a solgel (Ormocer 4) and a polymer (SU8). For a range of angles (up to $70^{\circ}$ from normal), and for low-energy $(<20 \mu \mathrm{J}), 40 \mathrm{~ns}$ pulses at $266 \mathrm{~nm}$ wavelength, the ablation depth along the direction of the incident laser beam is found to be independent of the angle of incidence. This allows the crater profiles at oblique incidence to be generated directly from the crater profiles at normal incidence by a simple coordinate transformation. This result is of use in the development of simulation tools for direct-write laser ablation. A simple model based on the moving ablation front approach is shown to be consistent with the observed behavior. (C) 2004 American Institute of Physics. [DOI: 10.1063/1.1655702]
\end{abstract}

Direct-write laser ablation using a focused laser beam is a highly promising technique for micromachining applications. Diode-pumped solid-state lasers have recently been employed for this purpose. This technique is particularly attractive for prototyping because it allows direct and rapid transfer from computer to workpiece without requiring an expensive mask. However, improved understanding of the fundamental ablation processes involved is required if this approach is to become more widely established. Direct-write micromachining involves the scanning of the focused laser beam over the surface of the workpiece along a predefined tool path, and overlap of multiple laser pulses on the surface is usually required to achieve the desired structure. In order to simulate arbitrary processes of this type and to predict their final surface shapes, it is necessary to understand the ablation of surfaces at nonnormal incidence. To investigate this aspect, we have studied the ablation behavior at varying angles of incidence in two interesting materials: a solgel (Ormocer 4) and a polymer photoresist (SU8). Our study is confined to the angular dependence of the ablation depth due to single laser pulses incident on a previously unablated surface; material changes in the workpiece arising from multipulse ablation (such as increased surface roughness and incubation effects) are not considered.

The samples consisted of thin $(\sim 100 \mu \mathrm{m})$ films of the solgel material (Ormocer 4) deposited on a borofloat glass substrate and SU8 polymer resist spun on a silicon wafer. The laser system (frequency quadrupled $\mathrm{Nd}: \mathrm{VO}_{4}$ ) produced 266-nm-wavelength, $Q$-switched pulses $<40 \mathrm{~ns}$ in duration, with a pulse energy ranging from 10 to $100 \mu \mathrm{J}$ measured at the laser. It had an $M^{2}$ value $<1.3$, beam divergence $<0.3$ mrad, diameter $\sim 2.5 \mathrm{~mm}$, and polarization $>100: 1$ horizontal. The repetition rate for most measurements was set to 20 $\mathrm{kHz}$. The laser output was expanded $\times 3$ and stopped down with an iris of about $7 \mathrm{~mm}$ diameter. After the scanning mirror, the beam was focused down using an $f \theta$ lens ( $f$ $=160 \mathrm{~mm}$ ). The beam was scanned at a sufficient speed to ensure the pulses were separated on the sample surface. The angle of incidence could be varied from $0^{\circ}$ to $90^{\circ}$ by rotating the sample on a goniometer stage. The focal spot was approximately $10 \mu \mathrm{m}$ in diameter with a depth of focus $\sim 350 \mu \mathrm{m}$. Craters were formed using different pulse energies, angles of incidence, and states of polarization. The samples were measured with a Zygo New View 200 surface profiler, based on scanning white light interferometry. In addition, scanning electron microscope (SEM) images of the samples were analyzed to provide more information about the surface detail and crater characteristics.

Typical SEM images of the craters $(\sim 10 \mu \mathrm{m}$ in diameter) are shown in Fig. 1. The craters had a maximum depth up to $\sim 3 \mu \mathrm{m}$. The sol-gel appears to have a rougher surface than the SU8 after ablation, particularly at pulse energies in excess of $30 \mu \mathrm{J}$. It also has a tendency to crack at higher energies. The rough surface may be due to local absorption by organic groups causing highly concentrated ablation points in the hybrid material. The SU8 generally shows a smoother surface profile, although at higher energies, the craters in SU8 show ripples due to liquefaction. The craters produced at lower energies appeared more predictable, and were selected for more detailed analysis. Typical crater cross sections taken from the Zygo for $11 \mu \mathrm{J}$ pulse energy are shown in Fig. 2. Each graph shows two experimental profiles, one measured at normal incidence (solid line) and the other at oblique incidence (dashed line). These slices were taken through the deepest point of the three-dimensional pro-

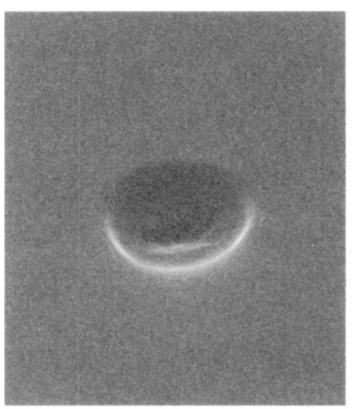

(a)

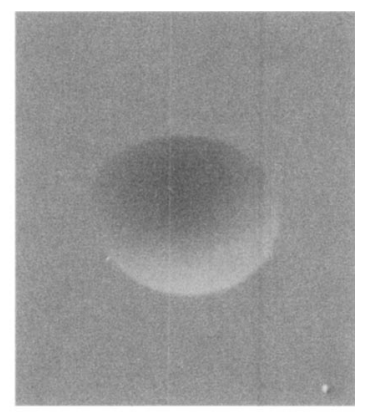

(b)
a)Electronic mail: a.holmes@imperial.ac.uk

FIG. 1. SEM images of craters. (a) Solgel $(11 \mu \mathrm{J})$ and (b) SU8 $(11 \mu \mathrm{J})$. 

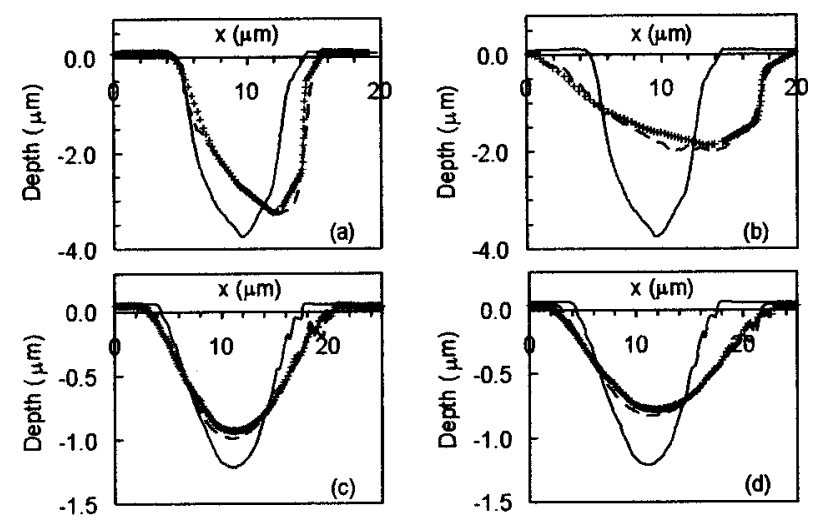

FIG. 2. Zygo crater cross sections in (a) solgel at $+30^{\circ}$, (b) solgel at $+60^{\circ}$, (c) SU8 at $-40^{\circ}$, and (d) SU8 at $-50^{\circ}$. Solid black line shows normal incidence; dashed line shows angled incidence (beam coming in from left of crater for solgel and right of crater for SU8); and crosses show fit to angled incidence.

file, in the plane of incidence; that is, the plane normal to the sample rotation axis. Note that the axes are not on the same scale.

For oblique incidence, the measured crater profiles became asymmetrical and were very similar for both polarizations over the range of angles investigated. Analysis of the results revealed that, in each case, close approximations to the crater profiles obtained at oblique incidence could be generated from the normal incidence profiles by a trivial coordinate transformation combining a shear and a rotation:

$$
\left(\begin{array}{l}
x \\
z
\end{array}\right)=\left(\begin{array}{cc}
-\sec \theta & -\sin \theta \\
0 & \cos \theta
\end{array}\right)\left(\begin{array}{l}
X \\
Z
\end{array}\right) \text {. }
$$

Here, $(x, z)$ and $(X, Z)$ are the coordinates of corresponding points on the oblique and normal incidence crater profiles, and $\theta$ is the angle of incidence for the oblique case (see Fig. 3 ). Each graph in Fig. 2 also shows (crosses) the oblique incidence profile generated using Eq. (1). The agreement between the measured and generated oblique incidence profiles is sufficiently close for discrepancies to be attributed to fluctuations in the beam. The observed behavior suggests that the ablation depth, if measured along the direction of the incoming laser beam (rather than normal to the sample surface), is invariant under rotation of the sample, and depends only on the transverse spatial intensity distribution of the beam.

The experimental results are consistent with the moving ablation front model used previously to describe laser ablation of polymers ${ }^{1-3}$ and metals. ${ }^{4,5}$ In the framework of this model, the ablation happens in a thin layer on the material surface. The surface propagates during the laser pulse as a result of material loss, and the crater shape is the result of this propagation. The starting point for analysis is the onedimensional (1-D) heat diffusion equation

$$
\frac{\partial T}{\partial t}=D_{T} \frac{\partial^{2} T}{\partial z^{2}}-\frac{1}{c_{p} \rho} \frac{\partial J}{\partial z}+v \frac{\partial T}{\partial z},
$$

where $D_{T}, c_{p}$, and $\rho$ are the heat diffusivity, specific heat capacity, and density, respectively, and $T$ is the temperature rise above ambient. The coordinate system of Eq. (2) is attached to the material surface (the ablation front) and $v$ is the velocity of the front along the $z$ direction (normal to the surface). $J$ is the power flow per unit area in the $z$ direction Downloaded 10 Sep 2007 to 155.198.4.89. Redistribution subject to

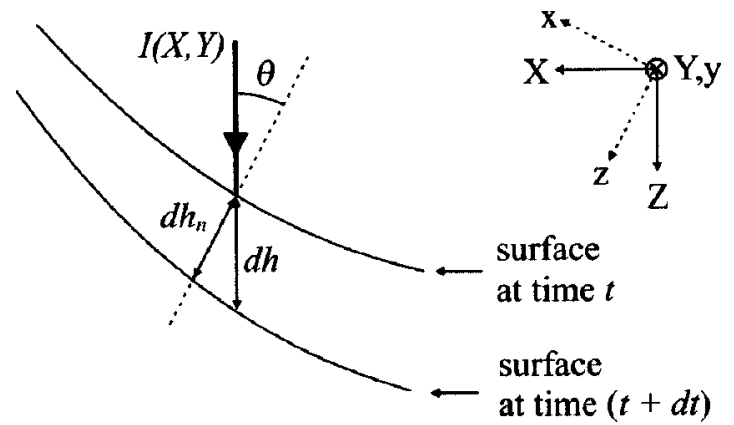

FIG. 3. Ablation model geometry.

associated with the light propagating inside the material. This is given by the $z$ component of the Poynting vector, which for normal incidence reduces to the local light intensity inside the material. The use of a 1-D model for heat flow is justified provided the beam diameter on the surface far exceeds the characteristic heat diffusion length of the solid. This is usually true for excimer laser ablation, as considered in Ref. 5. The approximation is also reasonable in our case, since the smaller size of the ablated spot (the beam diameter $\sim 10 \mu \mathrm{m}$ ) is compensated by lower thermal diffusion in polymers and glasses.

By solving Eq. (2) in combination with a model for thermally activated decomposition of the material, previous workers have shown that, for laser pulses with durations $\tau_{p}$ in the range of several tens of nanoseconds, the photothermal ablation process is characterized by two distinct phases separated by some critical point in time $\tau_{\mathrm{cr}} \cdot{ }^{1}$ During the initial phase, $0<t<\tau_{\mathrm{cr}}$, the surface temperature, $T_{s}=\left.T\right|_{z=0}$, rises until it reaches the temperature for material mass loss. During this phase, $v=0$. The ablation happens mainly during the second phase, $\tau_{\mathrm{cr}}<t<\tau_{p}$, when $T_{s}$ varies more slowly and the ablation front moves with the velocity $v>0$. The velocity falls sharply for $t>\tau_{p}$, terminating the process with little further material loss.

For the second phase, if the temperature distribution $T(z, t)$ is assumed to be in steady state, then Eq. (2) becomes stationary. ${ }^{2}$ In this case, taking into account the boundary conditions at the surface, $\partial T /\left.\partial z\right|_{z=0}=0$, and in the bulk, $\left.T\right|_{z \rightarrow \infty} \rightarrow 0,\left.J\right|_{z \rightarrow \infty} \rightarrow 0$, Eq. (2) may be integrated to obtain the velocity of the ablation front as

$$
v=\frac{J_{s}}{c_{p} \rho T_{s}} .
$$

Here, $J_{s}$ is the power flow per unit area just below the surface, which is related to the incident laser intensity $I_{0}$ by

$$
J_{s}=(1-R) I_{0} \cos \theta,
$$

where $R$ is the (angle dependent) reflectivity of the material. $J_{s}$ is generally a function of the lateral spatial coordinates, but the spatial variation is assumed to be slow and is neglected in Eqs. (2) and (3). This is consistent with the assumed 1-D model for $T(z, t)$. The same assumption is made in Eq. (4), allowing the usual plane wave reflectivity to be used.

With these approximations we can write that, during a small time interval $d t$, each point on the ablation front

propagates by a small amount:

AIP license or copyright, see http://apl.aip.org/apl/copyright.jsp 


$$
d h_{n}=v d t=\frac{I_{0}(1-R) \cos \theta}{c_{p} \rho T_{s}} d t,
$$

where the subscript $n$ denotes that the $d h_{n}$ is in the direction of the normal to the surface. From Fig. 3, the change in crater depth along the direction of the incident laser beam is given by $d h=d h_{n} / \cos \theta$, which can be written with the aid of Eq. (5) as

$$
d h=\frac{I_{0}(1-R)}{c_{p} \rho T_{s}} d t .
$$

It is important to note that the factor $\cos \theta$ in Eq. (5) does not appear in Eq. (6), making the incremental ablation depth $d h$ virtually angle independent. Some small degree of angular dependence might be expected for higher angles of incidence because of the reflectivity term. In general, two effects may occur: a uniform scaling of the crater depth as a result of the oblique illumination, and a modification to the normalized depth profile due to changes in the local angle of incidence as the crater develops. However, for shallow craters formed in materials with low real refractive index, as in our investigation, both of these effects are expected to be very weak except near grazing incidence, particularly if there are fluence-dependent effects that further reduce the reflectivity and effective refractive index below their normal values. Such effects have been reported previously by Hodapp and Fleming, ${ }^{6}$ who observed significant reductions in the reflectivity of polyimide during 248-nm-wavelength excimer laser ablation at oblique incidence.

Neglecting local variations in the angle of incidence during ablation, the total ablation depth $h$ along the direction of the incident laser beam may be obtained by integration of Eq. (6):

$$
\begin{aligned}
h(X, Y) & =\frac{(1-R)}{c_{p} \rho T_{s}} \int_{\tau_{\mathrm{cr}}}^{\tau_{p}} I_{0}(X, Y, t) d t \\
& =\frac{1}{\alpha} \frac{\left[F(X, Y)-F_{\mathrm{cr}}(X, Y)\right]}{F_{0}}, \quad F \geqslant F_{\mathrm{cr}},
\end{aligned}
$$

where

$$
\begin{aligned}
& F(X, Y)=\int_{0}^{\tau_{p}} I_{0}(X, Y, t) d t, \\
& F_{\mathrm{cr}}(X, Y)=\int_{0}^{\tau_{c r}} I_{0}(X, Y, t) d t, \quad F_{0}=\frac{c_{p} \rho T_{s}}{\alpha(1-R)},
\end{aligned}
$$

and $\alpha$ is the optical absorption constant of the material. In deriving Eq. (7), we have neglected transient effects at the start of the second phase, as predicted by more sophisticated models, ${ }^{1}$ and any material loss after the end of the laser pulse. Note that the transverse spatial variation of the laser fluence and intensity are now shown explicitly. The quantity $F_{\text {cr }}$ is the portion of the laser fluence that goes to initially raising the surface temperature to $T_{s}$. It is easily shown that, if the diffusion term in Eq. (2) is small, then $F_{\mathrm{cr}}=F_{0}$ at normal incidence, and Eq. (7) reduces to $h=\alpha^{-1}\left[\left(F / F_{0}\right)\right.$ $-1]$, implying a linear ablation curve. This kind of ablation characteristic is well known from work on excimer laser ablation. $^{6,7}$

When considering the possible angular dependence of the total ablation depth $h$, we can ignore the effect of reflectivity variations (that affect $h$ through the term $F_{0}$ ) except at extreme angles, for reasons already discussed in the context of the incremental depth. In addition, the effects of any angular dependence in $F_{\text {cr }}$ will be small except in regions of the crater where the fluence is close to the ablation threshold. Consequently, we can expect the total ablation depth, and hence the total volume of material removed by each pulse, to show negligible angular dependence at modest pulse energies. This is in agreement with our experimental results.

We expect that the theoretical model described here will show disagreement with experiment for larger fluence values, because the simplified photothermal ablation model will break down, and the increased crater depth will lead to significant changes in the angle of incidence as the crater evolves. A more rigorous model for the ablation process, coupled with a propagation algorithm that includes local variations in angle, has to be considered for this higher fluence regime. Poor agreement is also expected at very low pulse energies, where the fluence is close to $F_{\text {cr }}$ over a large portion of the crater. In this regime, the angular dependence of $F_{\text {cr }}$ will tend to reduce the ablation depth and volume of material removed at oblique incidence.

We have investigated the behavior of focused laser ablation for single laser pulses at varying angles of incidence in a solgel (Ormocer 4) and a polymer (SU8). We have shown experimentally that, under certain conditions, the ablation depth along the direction of the incident laser beam is independent of angle of incidence. This allows crater profiles at angled incidence to be generated directly from the crater profiles at normal incidence by a simple coordinate transformation. The observed behavior is consistent with a simple model for the ablation process based on the moving ablation front approach. The result will be useful in the prediction of oblique incidence ablation for the controlled production of more complex structures.

The authors thank Frank Otte at Laser Zentrum Hannover for his help with this work and the European Commission for supporting this project.

\footnotetext{
${ }^{1}$ N. Arnold, N. Bityurin, and D. Bauerle, Appl. Surf. Sci. 138-139, 212 (1999)

${ }^{2}$ N. Bityrin, N. Arnold, B. Luk'yanchuk, and D. Bauerle, Appl. Surf. Sci. 127-129, 164 (1998).

${ }^{3}$ B. Luk'yanchuk, N. Bityurin, M. Himmelbauer, and N. Arnold, Nucl. Instrum. Methods Phys. Res. B 122, 347 (1997).

${ }^{4}$ S. Kuper, K. Brannon, and J. Brannon, J. Appl. Phys. 76, 1203 (1994).

${ }^{5}$ J. H. Brannon and K. W. Brannon, J. Vac. Sci. Technol. B 7, 1275 (1989).

${ }^{6}$ T. W. Hodapp and P. R. Fleming, J. Appl. Phys. 84, 577 (1998).

${ }^{7}$ J. R. Sobehart, J. Appl. Phys. 74, 2830 (1993).
} 